\title{
Plasma interleukin-18 levels are a biomarker of innate immune responses that predict and characterize tuberculosis-associated immune reconstitution inflammatory syndrome
}

\author{
Hong Yien Tan ${ }^{a}$, Yean Kong Yong ${ }^{a}$, Bruno B. Andrade ${ }^{b}$, \\ Esaki M. Shankar ${ }^{\mathrm{a}, \mathrm{c}}$, Sasheela Ponnampalavanar ${ }^{\mathrm{a}, \mathrm{d}}$, \\ Sharifah F.S. Omar, ${ }^{a, d}$, Gopalan Narendran ${ }^{\mathrm{e}}$, \\ Adeeba Kamarulzaman ${ }^{\mathrm{a}, \mathrm{d}}$, Soumya Swaminathan ${ }^{\mathrm{e}}$, Irini Sereti ${ }^{\mathrm{b}}$, \\ Suzanne M. Crowe ${ }^{f, g, h}$ and Martyn A. French ${ }^{i, j}$
}

Background: Tuberculosis-associated immune reconstitution inflammatory syndrome (TB-IRIS) is a substantial problem in HIV/TB coinfected patients commencing antiretroviral therapy (ART). The immunopathogenesis of TB-IRIS includes increased production of proinflammatory chemokines and cytokines, including interleukin-18, which is a signature cytokine of the nucleotide-binding domain and leucine-rich repeat pyrin containing protein-3 inflammasome. We compared plasma levels of interleukin18 and other biomarkers of monocyte/macrophage activation in the prediction and characterization of TB-IRIS.

Methods: Biomarkers were assayed pre-ART and during TB-IRIS, or equivalent timepoint, in a case-control study of Malaysian HIV patients with paradoxical or unmasking TB-IRIS $(n=15)$, TB no IRIS $(n=14)$, and no TB or IRIS $(n=15)$. Findings for interleukin18 were verified in another cohort of patients with paradoxical TB-IRIS $(n=26)$ and their controls $(n=22)$ from India.

Results: Interleukin-18 was higher in TB-IRIS patients pre-ART and during the event in both Malaysian patients $(P<0.0001)$ and Indian patients $(P<0.01)$. CXCL10 was higher pre-ART $(P<0.001)$, mainly in paradoxical TB-IRIS patients, and during TB-IRIS $(P<0.001)$, whereas CXCL8 was only higher during TB-IRIS $(P<0.001)$. Soluble(s) CD14 was increased in all patients with HIV/TB coinfection pre-ART and during TB-IRIS or equivalent time-point, compared with patients without TB. In contrast, interferon- $\gamma$ was lower before and during TB-IRIS. By receiver operating curve analysis, CXCL10, and/or interleukin-18 pre-ART were predictive of TB-IRIS.

Conclusion: Plasma interleukin-18 levels pre-ART are candidate biomarkers for predicting paradoxical and unmasking TB-IRIS and should be investigated for risk stratification and elucidation of disease pathogenesis.

(c) 2015 Wolters Kluwer Health, Inc. All rights reserved.

\footnotetext{
${ }^{a}$ Faculty of Medicine, Centre of Excellence for Research in AIDS (CERiA), University of Malaya, Kuala Lumpur, Malaysia, ${ }^{\mathrm{b}}$ National Institute of Allergy and Infectious Diseases, National Institutes of Health, Bethesda, Maryland, USA, ${ }^{\mathrm{C}}$ Faculty of Medicine, Tropical Infectious Diseases Research and Education Centre (TIDREC), Department of Medical Microbiology, dInfectious Disease Unit, Department of Medicine, University of Malaya Medical Centre, Kuala Lumpur, Malaysia, eNational Institute for Research in Tuberculosis, Chennai, India, ${ }^{f}$ Centre for Biomedical Research, Macfarlane Burnet Institute for Medical Research and Public Health, IInfectious Diseases Unit, The Alfred Hospital, h'Department of Infectious Diseases, Monash University, Melbourne, ${ }^{i}$ School of Pathology and Laboratory Medicine, University of Western Australia, and ${ }^{\mathrm{j}}$ Department of Clinical Immunology, Royal Perth Hospital and PathWest Laboratory Medicine, Perth, Western Australia, Australia.

Correspondence to Professor Martyn A. French, School of Pathology and Laboratory Medicine, University of Western Australia, Level 2, MRF Building, Rear, 50 Murray Street, Perth, WA 6000, Australia.
}

E-mail: martyn.french@uwa.edu.au

Received: 13 September 2014; revised: 23 November 2014; accepted: 3 December 2014.

ISSN 0269-9370 Copyright (c) 2015 Wolters Kluwer Health, Inc. All rights reserved. Copyright @ 2015 Wolters Kluwer Health, Inc. Unauthorized reproduction of this article is prohibited. 
AIDS 2015, 29:000-000

\section{Keywords: antiretroviral therapy, CXCL10, HIV, interferon- $\gamma$, interleukin-18, interleukin-18 binding protein, sCD14, tuberculosis-associated immune reconstitution inflammatory syndrome, tuberculosis}

\section{Introduction}

Tuberculosis (TB) is the most common opportunistic infection among individuals with HIV infection, especially in resource-limited countries. The use of antiretroviral therapy (ART) has reduced morbidity and mortality in HIV-infected patients but may be complicated by an immune reconstitution inflammatory syndrome (IRIS) in patients who commence ART with a low $\mathrm{CD}^{+}$T-cell count [1]. Following initiation of ART, $10-30 \%$ of TB-HIV coinfected patients experience a paradoxical deterioration of treated TB, or a rapid onset of newly diagnosed TB associated with an exaggerated inflammatory response, conditions that are referred to as paradoxical TB-IRIS and unmasking TBIRIS, respectively [2]. As TB-IRIS usually occurs within the first month of ART, it can be difficult to distinguish from relapsed or newly acquired TB. Corticosteroid therapy is effective in patients who experience severe TBIRIS [3]; however, the potential for adverse effects limit its use. Therefore, there is an urgent need for laboratory markers to predict and identify TB-IRIS $[3,4]$.

Risk factors for the development of an IRIS in HIVinfected patients are reported to be a low $\mathrm{CD}^{+}{ }^{+} \mathrm{T}$-cell count at ART initiation [5], robust immunological and virological response to ART [6], high antigenic burden of an underlying opportunistic infection at ART initiation [7], and early initiation of ART after opportunistic infection treatment $[6,8]$. However, the immunological mechanisms underlying the development of an IRIS are not clearly understood. Several studies have demonstrated an expansion of Mycobacterium tuberculosis-specific interferon-gamma (IFN- $\gamma$ ) producing $T$ cells $[9,10]$ or elevation of polarized T-helper 1 (Th1) responses [11] among TB-IRIS patients. However, these responses may also occur in non-IRIS patients [12,13], questioning their relevance.

Accumulating evidence suggests that innate immune responses play a role in the pathogenesis of TB-IRIS [14-16]. A recent microarray investigation of RNA from monocytes of patients with TB-IRIS demonstrated that 100 genes related to 'inflammatory disease,' 'immunological disease,' 'cellular movement,' 'hematological system development and function,' and 'immune cell trafficking' were perturbed at least 1.5 -fold in patients relative to controls [17]. These findings suggest that monocytes contribute to the pathogenesis of TB-IRIS.
Given the strong interplay between innate and adaptive immune responses, the onset of TB-IRIS may stem from protective immune responses that are excessive in magnitude resulting from dysregulation of the interaction between innate and adaptive immune responses [16].

Here, we sought to identify and compare plasma biomarkers of innate immune responses that predict and characterize TB-IRIS, including interleukin-18, which is increased in TB-IRIS patients [14] and is a signature cytokine of the nucleotide-binding domain and leucine-rich repeat pyrin containing protein-3 (NLRP3) inflammasome [18]. We established that elevated plasma levels of interleukin-18 and CXCL10 predicted TB-IRIS and that levels of IL-18BPa, the most abundant splice variant of interleukin-18 binding protein (IL-18BP) that regulates the biological activity of interleukin-18 [19], may not be sufficient to regulate the biological functions of interleukin-18. These findings suggest that TB-IRIS is associated with an exaggerated inflammatory response that includes increased interleukin-18 production, which may be inadequately regulated.

\section{Methods}

\section{Study groups and design}

The study was initially conducted on samples from patients that were selected from a prospective observational cohort study of $200 \mathrm{HIV-infected} \mathrm{ART-naive}$ adults (>18 years) initiating ART between 2005 and 2012 with a CD4 ${ }^{+}$T-cell count of less than 200 cells $/ \mu l$ at the University Malaya Medical Centre (UMMC), Malaysia (Kuala Lumpur cohort) [20]. Symptomatic patients were screened for TB by chest radiograph and/or sputum examination and those with TB completed a median of 5 weeks (range 1-20 weeks) of antituberculous therapy (ATT) prior to ART initiation. Participants were reviewed 4, 12, 24, and 48 weeks after ART initiation. Blood samples were collected at each follow-up visit and additional samples were collected from those who developed symptoms suggestive of paradoxical or unmasking TB-IRIS. Informed consent was obtained from all participants and the study was approved by the Medical Ethics Committee of the UMMC (MEC Ref. No: 673.33).

We excluded patients who were not adherent to ATT or ART, nonresponders due to ATT or ART drug resistance, 
and those who developed malignancy or multiple diagnoses of an IRIS. Paradoxical and unmasking TB-IRIS were later classified by an expert panel, which was blinded from test results, according to diagnostic criteria of the International Network for the Study of HIV-associated IRIS [21]. Demographic and clinical information on patients are listed in Supplementary Table 1, http://links.lww.com/QAD/A626. All cases wherein plasma samples were available for both baseline (within 2 weeks before ART initiation) and TB-IRIS (within 2 weeks of symptom onset), or equivalent time-point (EQT) in controls, were included. Controls were matched 2:1 to TB-IRIS cases based on baseline TB status, age, and $\mathrm{CD} 4^{+} \mathrm{T}-$ cell counts (within 50 cells/ $\mu \mathrm{l}$ ).

Participants were classified into three groups according to the following definitions [22]:

(1) TB-IRIS (case): HIV-infected patients who had TB prior to initiation of ART and were treated with ATT, who subsequently experienced disease classified as TBIRIS (paradoxical TB-IRIS); or HIV-infected patients who had no evidence of TB prior to initiation of ART and subsequently presented with TB that exhibited exaggerated inflammation after commencing ART (unmasking TB-IRIS).

(2) TB no IRIS (control): HIV-infected individuals who had TB prior to initiation of ART and were treated with ATT, with no clinical deterioration after commencing ART.

(3) No TB or IRIS (control): HIV-infected individuals who did not have TB prior to initiation of ART and did not develop TB-IRIS after commencing ART.

To confirm the findings for interleukin-18, samples were analysed from patients selected from a randomized controlled clinical trial (NCT 933790) conducted at the National Institute for Research in Tuberculosis, Chennai, India (Chennai cohort) [23,24]. Fifty-seven patients with newly diagnosed sputum culture-confirmed pulmonary TB were enrolled. Patients $(n=48)$ were selected based on the criteria described elsewhere [25]. Baseline investigations were repeated at event in the paradoxical TB-IRIS group $(n=26)$ and at week 6 of ART in patients who had uneventful ART initiation $(n=22)$.

\section{Laboratory investigations}

In the Kuala Lumpur cohort, serum/plasma levels of neopterin (RE59321; IBL International $\mathrm{GmBH}$ ); sCD163, sCD14, IFN- $\gamma$ (DC1630, DC140, and DIF50; R\&D Systems); interleukin-6, interleukin-10, tumor necrosis factor (TNF) $\alpha$, CCL2, CXCL8, CXCL10 (550799, 550613, 550610, 559017, 550999, and 550926; BD Biosciences) were measured by ELISA. The interleukin-18 ELISA kits consisted of recombinant human interleukin-18, primary and secondary antibodies (B001-5, D044-3, and D045-6; R\&D Systems). The assay was performed on Nunc Maxisorp 96-well plates (62407-162; Thermo Scientific). Ferritin levels were measured in the clinical diagnostic laboratory of UMMC. IL-18BPa was assayed by ELISA (DBP180, R\&D Systems). All analytes were assayed according to manufacturers' protocols. The bioavailability of free circulating interleukin-18 (fraction of cytokine not bound to its inhibitor, IL-18BP) was estimated using the law of mass action [26]. Briefly, bioavailability of interleukin-18 was calculated using the molecular weights of interleukin-18 (18.4 kDa) and IL-18BP (17.6 kDa) and their interaction at a ratio of $1: 1$ with dissociation constant $\left(K_{\mathrm{d}}\right)$ of $0.4 \mathrm{nmol} / 1$ [27]:

$K_{\mathrm{d}}=\frac{[\text { Ligand }] \cdot[\text { Receptor }]}{[\text { Ligand }] \cdot[\text { Receptor }]}$

and was applied as follows:

$x=\frac{-b+\sqrt{b^{2}-4 c}}{2}$

where $\chi$ is [interleukin-18 $]_{\text {free }}, b$ is $[$ IL-18BP] $-[$ interleukin-18] $+K_{\mathrm{d}}, c$ is $-K_{\mathrm{d}} \bullet$ [interleukin-18] and the molar concentration of the analyte [28].

In the Chennai cohort, interleukin-18 was assayed in cryopreserved EDTA-treated plasma using an ELISA kit from R\&D systems (7620), following the manufacturer's instructions.

\section{Statistical analysis}

The primary analysis was to compare biomarkers among the three groups of patients before ART and at the time of TB-IRIS or EQT in controls. Comparisons of categorical variables were tested using chi-square test or Fisher's exact test, whereas continuous variables were tested using the nonparametric Kruskal-Wallis test for multiple group comparisons. If significant differences were seen, each marker was tested separately using Mann-Whitney $U$ tests applying Bonferroni correction. The predictive power of pre-ART levels of biomarkers was examined using receiver operating characteristic (ROC) analysis. A cut-off value was obtained from ROC analysis and the odds ratio for predicting TB-IRIS was calculated using a binary regression model. Correlations were estimated using Spearman's test. Statistical analyses were performed using SPSS, version 20 and Prism, version 5.02 (GraphPad) softwares. Statistical significance was defined as $P<0.05$ except in analyses that included a Bonferroni correction where a $P$ value of 0.0042 was considered to be significant. 


\section{Results}

\section{Baseline demography}

The pre-ART characteristics for the Kuala Lumpur cohort patients categorized as TB-IRIS $(n=15)$ [comprising paradoxical TB-IRIS $(n=9)$ and unmasking TB-IRIS $(n=6)]$, TB no IRIS $(n=14)$, and no TB or IRIS $(n=15)$ are shown in Supplementary Table 1, http://links.lww.com/QAD/A626. We observed that the three patient groups had similar pre-ART $\mathrm{CD}^{+}{ }^{+}$T-cell counts $(P=0.189), \mathrm{CD}^{+}$T-cell percentages $(P=0.253)$, age $(P=0.384)$, and HIV viral load $(P=0.739)$. Furthermore, no significant difference was observed in the interval from ATT to ART initiation between patients with paradoxical TB-IRIS and the TB no IRIS controls $(P=0.493)$. However, there was a significant difference in the form of TB disease at baseline between TB-IRIS and TB no IRIS patients $(P<0.001)$, whereby disseminated TB was more prevalent among TB-IRIS patients (66.7\%) as compared with TB no IRIS patients (33.3\%).

\section{Increased plasma levels of interleukin-18 and decreased levels of interferon- $\boldsymbol{\gamma}$ prior to initiation of antiretroviral therapy were consistently associated with tuberculosis- associated immune reconstitution inflammatory syndrome}

Comparison of all three study groups in the Kuala Lumpur cohort revealed that patients experiencing TB-IRIS exhibited higher plasma interleukin-18 levels pre-ART compared with the TB no IRIS or the no TB or IRIS controls (both $P<0.0001$ ) (Fig. 1a-e). During the TBIRIS event (one sample was obtained per patient, ranging from 29 to 75 days post ART initiation), levels of interleukin-18 remained higher in comparison with control groups $(P=0.0001$ and $<0.0001$, respectively) (Fig. $2 \mathrm{a}-\mathrm{e})$.

As compared with the TB no IRIS or the no TB or IRIS controls, TB-IRIS patients also displayed lower plasma levels of IFN- $\gamma$ both pre-ART $(P=0.003$ and $<0.0001$, respectively) and during TB-IRIS $(P=0.0008$ and $<0.0001$, respectively) (Figs $1 \mathrm{f}$ and $2 \mathrm{f}$ ). There was no difference in IFN- $\gamma$ levels between TB no IRIS controls compared with the no TB or IRIS controls at baseline and the event/EQT (Figs 1f and 2f). Together, we showed that increased plasma levels of interleukin-18 and decreased plasma levels of IFN- $\boldsymbol{\gamma}$ prior to ART were associated with the subsequent development of TB-IRIS.

\section{The development of tuberculosis-associated immune reconstitution inflammatory syndrome was associated with increased plasma levels of CXCL10 and CXCL8}

In view of the previous observation that plasma levels of the chemokines CXCL10 and CCL2 were different in HIV patients with TB-IRIS compared with TB/HIV patients who did not develop an IRIS [14], we next investigated if plasma levels of CXCL10, CCL2, or
CXCL8 were potential biomarkers for the prediction or diagnosis of TB-IRIS. Pre-ART plasma levels of CXCL10 were increased in TB-IRIS $(P=0.0002)$ (Fig. 1g). Further analysis of the data revealed that the higher CXCL10 levels were mostly in patients with paradoxical TB-IRIS (Supplementary Figure 1, http://links.lww.com/QAD/A626). During TB-IRIS, CXCL10 and CXCL8 were higher in TB-IRIS patients $(P<0.0001$ and $P<0.0001$, respectively) compared with controls (Fig. $2 \mathrm{~g}$ and k, respectively).

\section{Higher plasma levels of sCD14 were associated with HIV-tuberculosis coinfection}

To determine if other biomarkers of monocyte and macrophage activation had the potential to predict the onset of TB-IRIS, we also compared plasma levels of ferritin, neopterin, TNF- $\alpha$, interleukin- 6 , interleukin10 , soluble(s) CD163 and sCD14 in the different study groups. None of these biomarkers were predictive to TBIRIS (Figs 1a-d, h-1 and 2a-d, h-l). Although sCD163 and sCD14 levels were higher in TB-IRIS patients than no TB or IRIS controls, there were no significant differences between TB-IRIS and TB no IRIS controls. Of note, we observed that, compared with the no TB or IRIS controls, TB no IRIS controls also had higher levels of sCD14 at both pre-ART and EQT $(P<0.0001$ and $P<0.0001$, respectively) (Figs $1 \mathrm{~d}$ and $2 \mathrm{~d}$ ) These results suggest that increased plasma levels of sCD14 are tightly associated with HIV-TB coinfection.

\section{Plasma levels of interleukin-18 and CXCL10 prior to antiretroviral therapy initiation predicted the development of tuberculosis-associated immune reconstitution inflammatory syndrome}

Using ROC analyses, plasma levels of both interleukin-18 and CXCL10 prior to ART initiation were observed to be strong candidate biomarkers for predicting paradoxical TB-IRIS [area under curve $($ AUC) $=0.99, P<0.0001$ and $A U C=0.884, P<0.0001$, respectively] (Fig. 3a and b). The lower AUC observed for CXCL10 compared with interleukin-18 may reflect the observation that interleukin-18 levels were higher pre-ART in both paradoxical and unmasking TB-IRIS whereas CXCL10 levels were higher in only paradoxical TB-IRIS (Supplementary Figure 1, http://links.lww.com/QAD/ A626). In contrast, plasma levels of IFN- $\gamma$ were not predictive to TB-IRIS (Fig. 3c). Based on the ROC analysis, we determined that a cut-off value more than $8200 \mathrm{pg} / \mathrm{ml}$ for interleukin-18 was strongly predictive of TB-IRIS (odds ratio of 175; 95\% confidence interval $=14-216, P<0.0001)$.

Plasma levels of interleukin-18 binding protein may be inappropriately low in tuberculosisassociated immune reconstitution inflammatory syndrome patients

Having demonstrated that plasma levels of interleukin-18 were elevated two-fold in patients with both paradoxical 
(a) Ferritin

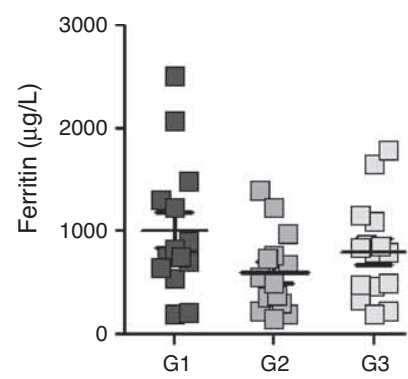

(d)

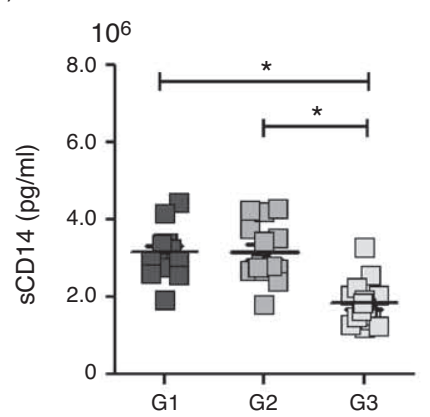

(g)

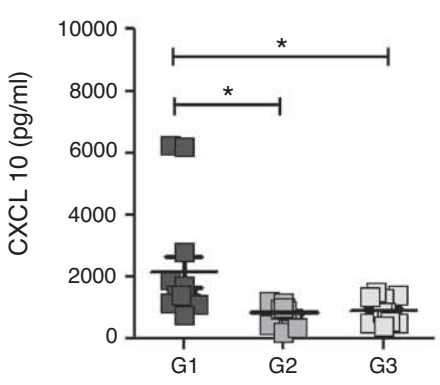

(j)

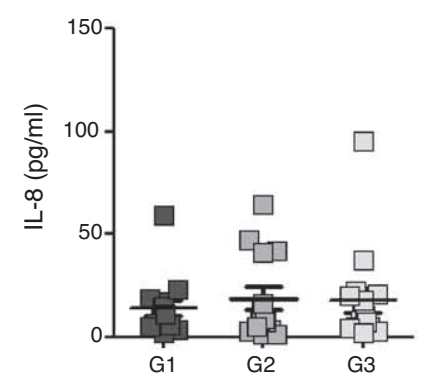

(b)

Neopterin

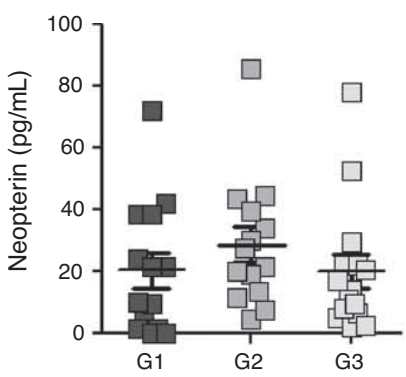

(e)

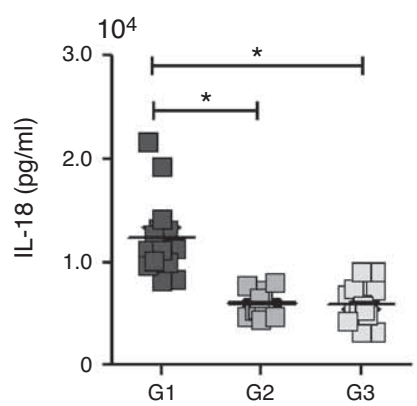

(h)

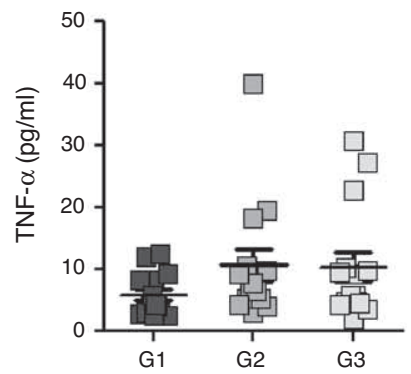

(k)

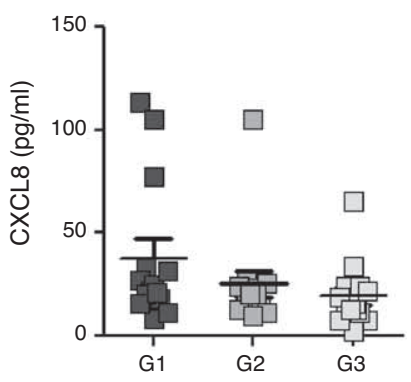

(c) $\quad \mathrm{SCD} 163$

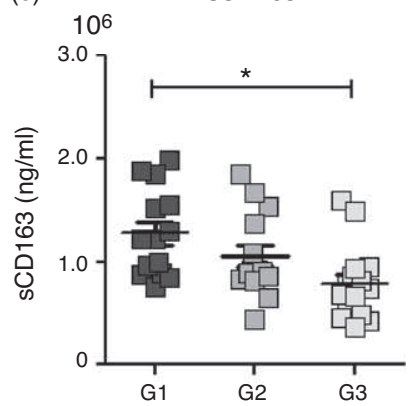

(f)

IFN- $\gamma$

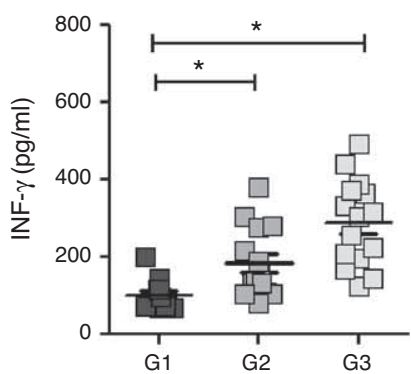

(i)

CCL-2

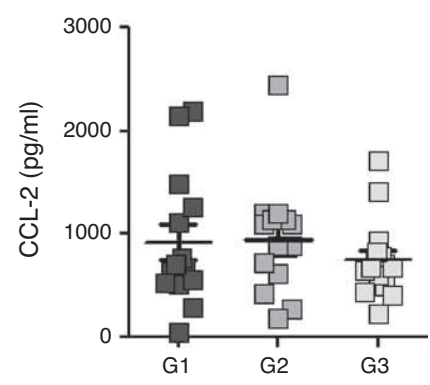

(I)

IL-10

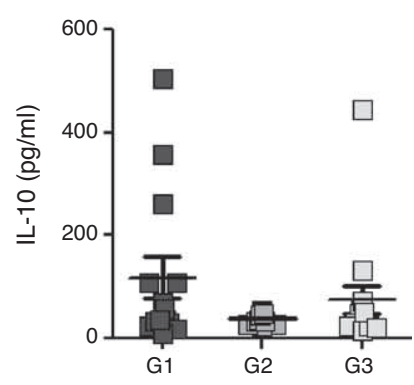

G1: TB-IRIS (Case)

$\square$ G2: TB no IRIS (Control)

$\square$ G3: No TB or IRIS (Control)

Fig. 1. Comparison of plasma levels of biomarkers in tuberculosis-associated immune reconstitution inflammatory syndrome patients and controls at baseline. (a) Ferritin, (b) Neopterin, (c) sCD163, (d) sCD14, (e) interleukin-18, (f) IFN- $\gamma$, (g) CXCL10, (h) TNF- $\alpha$, (i) CCL2, (j) interleukin-6, (k) CXCL8, (I) interleukin-10. Levels of biomarkers were compared across the three patient groups by Kruskal-Wallis test. Post hoc Mann-Whitney $U$ tests were then performed for those biomarkers with a Kruskal-Wallis test $P$ value of $<0.05$. $* P<0.0042$ (adjusted for multiple comparisons). IFN, interferon; TB-IRIS, tuberculosis-associated immune reconstitution inflammatory syndrome; TNF, tumor necrosis factor. 
(a)

Ferritin

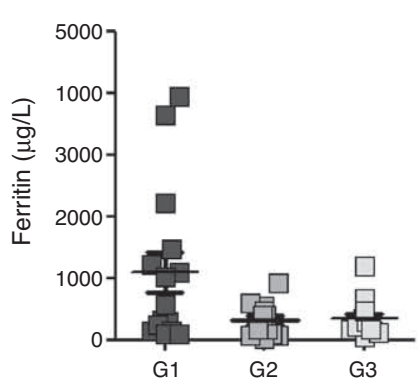

(d)

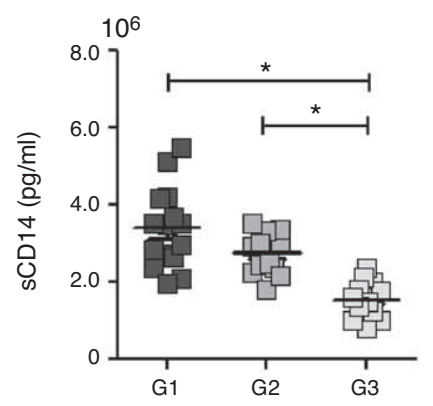

(g)

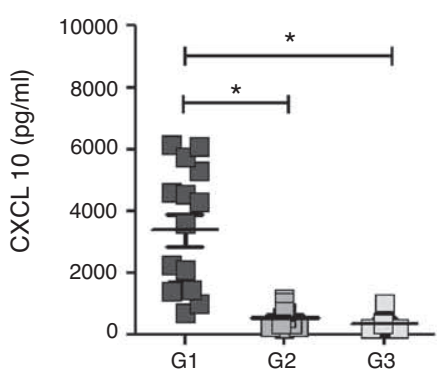

(j)

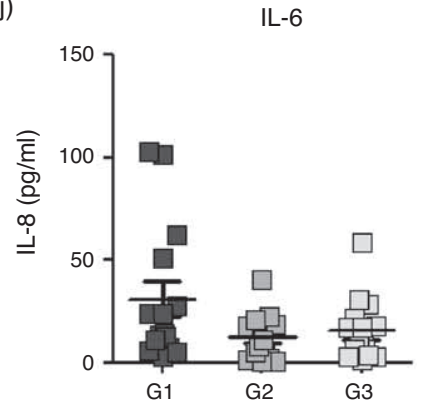

(b)

Neopterin

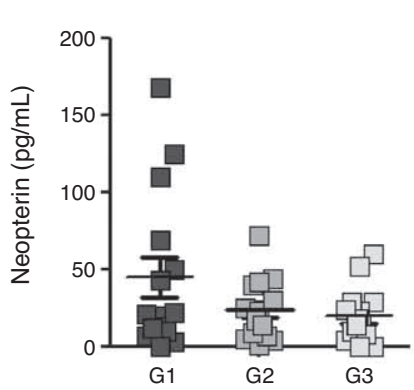

(e)

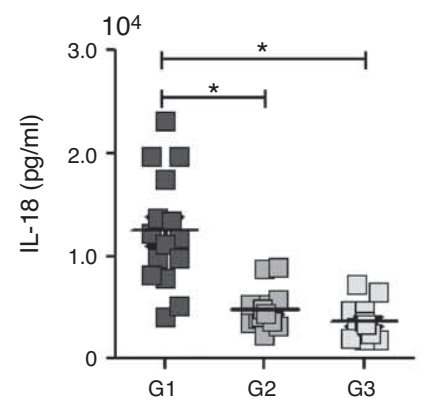

(h)

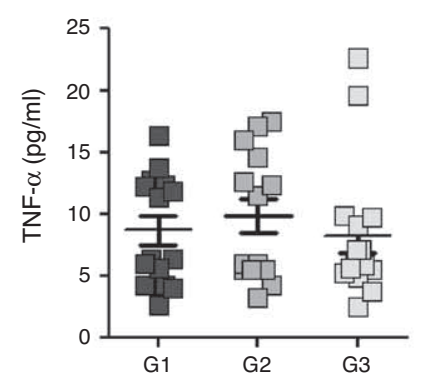

(k)

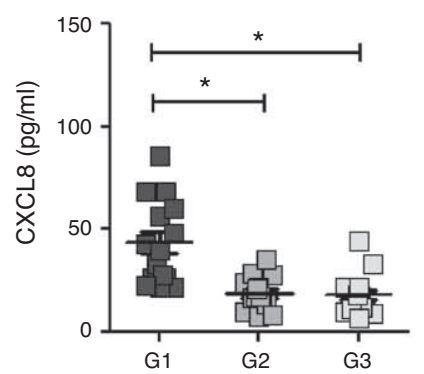

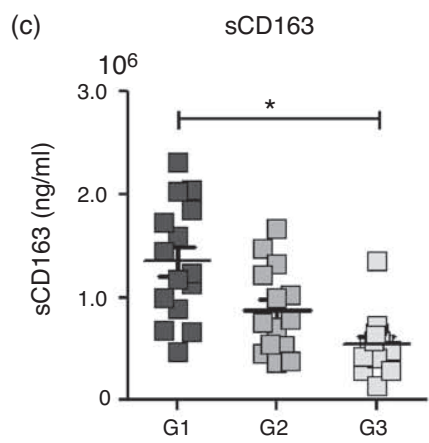

(f)

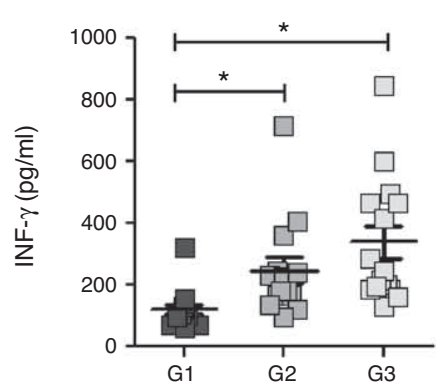

(i)

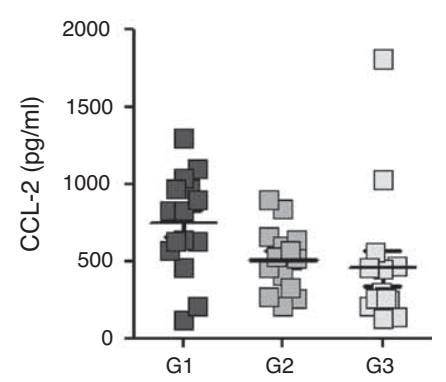

(I)

IL-10

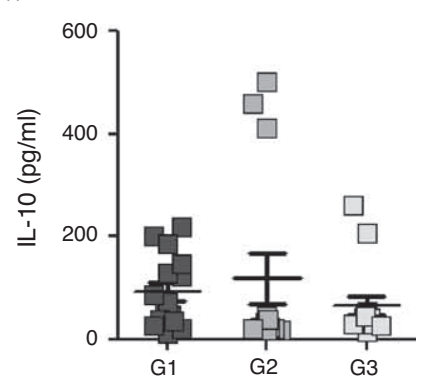

G1: TB-IRIS (Case)

G2: TB no IRIS (Control)

G3: No TB or IRIS (Control)

Fig. 2. Comparison of plasma levels of biomarkers in tuberculosis-associated immune reconstitution inflammatory syndrome patients and controls at clinical events. (a) Ferritin, (b) Neopterin, (c) sCD163, (d) sCD14, (e) interleukin-18, (f) IFN- $\gamma$, (g) CXCL10, (h) TNF- $\alpha$, (i) CCL2, (j) interleukin-6, (k) CXCL8, (I) interleukin-10. Levels of biomarkers were compared across the three patient groups by Kruskal-Wallis test. Post hoc Mann-Whitney $U$ tests were then performed for those biomarkers with a Kruskal-Wallis test $P$ value of $<0.05 . * P<0.0042$ (adjusted for multiple comparisons). IFN, interferon; TB-IRIS, tuberculosis-associated immune reconstitution inflammatory syndrome; TNF, tumour necrosis factor. 
(a)

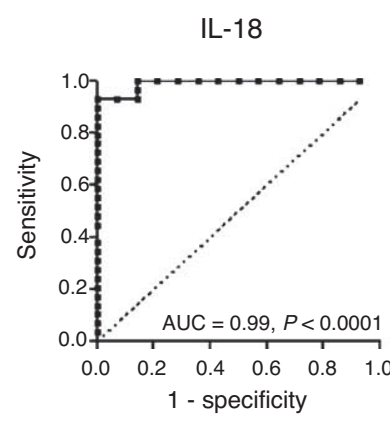

(b)

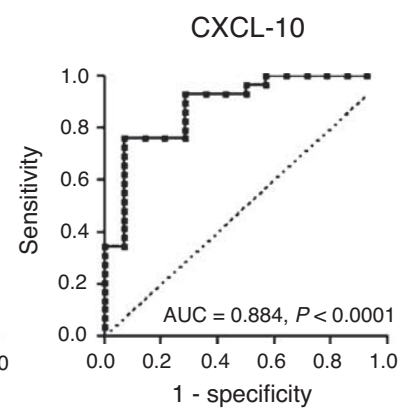

(c)

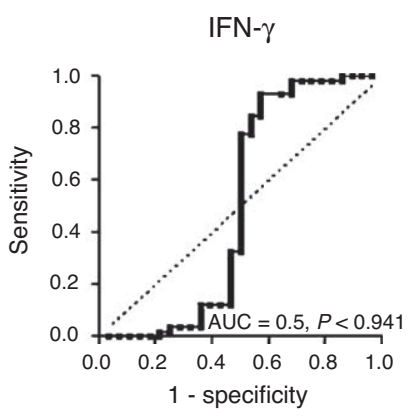

(d)

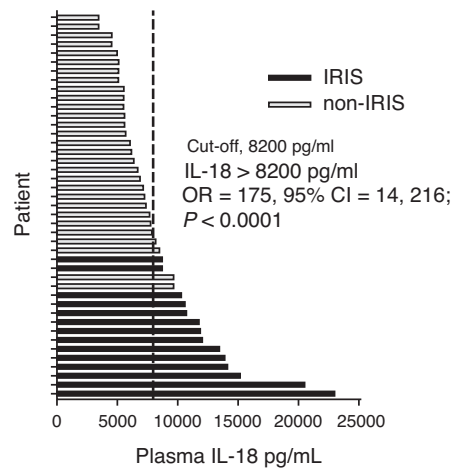

Fig. 3. Receiver operating characteristic curves for prediction of tuberculosis-associated immune reconstitution inflammatory syndrome (a) interleukin-18, (b) CXCL-10, (c) IFN- $\gamma$ and (d) Cut-off of interleukin-18 value that predicts TB-IRIS. AUC, area under curve; IFN, interferon; $\mathrm{Cl}$, confidence interval; $\mathrm{OR}$, odds ration; TB-IRIS, tuberculosis-associated immune reconstitution inflammatory syndrome.

and unmasking TB-IRIS both pre-ART and during TBIRIS, and given the role of interleukin-18 in the NLRP3 inflammasome [29], we hypothesized that interleukin-18, in particular, is linked to the immunopathogenesis of TBIRIS. As interleukin-18 activity is regulated by IL-18BP, a soluble molecule that binds mature interleukin-18 with high affinity and prevents its interaction with cell surface receptors, thus restricting the bioavailability of interleukin-18 [19], we next assessed the circulating levels of this regulatory molecule. We demonstrated that although TB-IRIS patients had higher plasma levels of IL-18BPa prior to ART initiation and during TB-IRIS, levels of free circulating interleukin-18 were higher in TB-IRIS patients compared with controls (Fig. 4a). ROC analysis showed that, like plasma interleukin-18 levels, free interleukin-18 levels were predictive of TB-IRIS $(\mathrm{AUC}=0.847, \quad P=0.00026 \quad$ (Fig. 4b). These observations suggest that the balance between interleukin-18 and IL-18BP in plasma may be critical in setting the stage for TB-IRIS by determining the bioavailability of free interleukin-18.

\section{Higher plasma interleukin-18 levels in paradoxical tuberculosis-associated immune reconstitution inflammatory syndrome were confirmed in the Chennai cohort}

To confirm the potential use of plasma interleukin-18 levels as a biomarker for predicting TB-IRIS, we assayed plasma levels of this cytokine in an independent cohort of patients from Chennai. Consistent with the results observed in the Kuala Lumpur patients, paradoxical TB-IRIS patients from Chennai exhibited higher plasma interleukin-18 levels pre-ART $(P=0.0043)$ and during the TB-IRIS event $(P=0.0004)$ compared with HIV/ TB patients who did not develop TB-IRIS (Fig. 5a). In addition, the ROC analysis also validated the observations in the Kuala Lumpur patients by demonstrating that plasma interleukin-18 levels predicted paradoxical TB-
IRIS (AUC $=0.742, P=0.004$ ) (Fig. 5b). A cut-off value of $520 \mathrm{pg} / \mathrm{ml}$ for interleukin-18 predicted TB-IRIS with an odds ratio of 3.3 (95\% confidence interval $=1-10$, $P=0.048$ ) (Fig. 5c). The predictive value was therefore lower than in the Kuala Lumpur cohort (Fig. 3d).

\section{Discussion}

There are currently no biomarkers that predict TB-IRIS in HIV/TB patients. Inflammation is a prominent feature of an IRIS, and monocytes/macrophages play a critical role in both inflammatory responses and control of M. tuberculosis infection. Thus, we investigated plasma biomarkers associated with inflammation, with a particular focus on monocytes/macrophages, in a case-control study of HIV/TB patients with either paradoxical or unmasking TB-IRIS. We demonstrated higher plasma interleukin-18 levels before ART and during TB-IRIS, when compared with controls, and confirmed this in an independent cohort of HIV-infected patients with TBIRIS. Furthermore, ROC analyses demonstrated that interleukin-18 levels before ART were the strongest candidate biomarker for predicting both paradoxical and unmasking TB-IRIS. However, differences in the plasma interleukin-18 level that predicted TB-IRIS, and the certainty with which this prediction could be made, were observed in the two cohorts of patients studied. This finding might reflect the use of different assay methods or differences in patient characteristics and requires further investigation.

Inflammation plays a pivotal role in defenses against infectious agents, but can become dysregulated and cause deleterious consequences to the host [30]. In the absence of effective T-cell responses, the immune system 'defaults' to innate immune responses and 'upstream' inflammatory markers produced by innate immune cells are particularly important as they govern the downstream inflammatory 
(a)
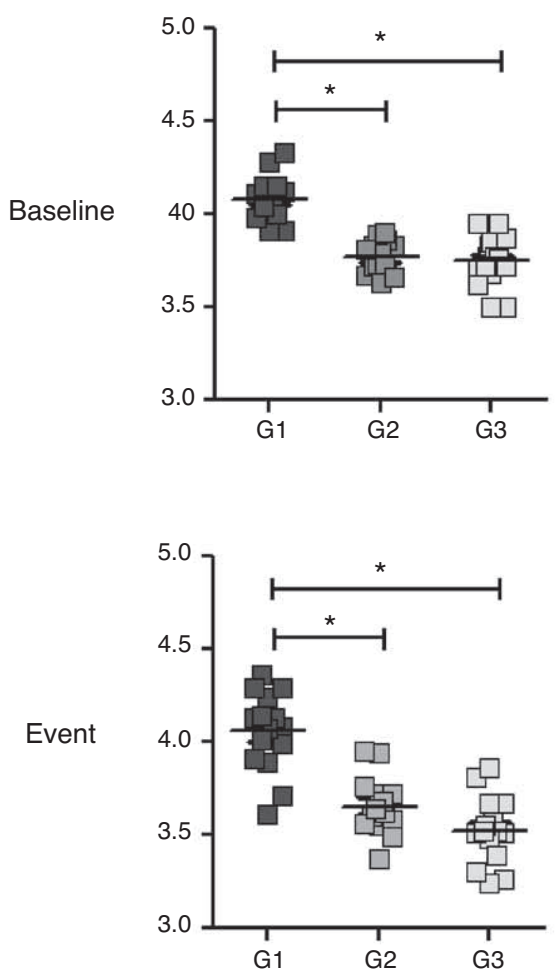

G1: TB-IRIS (Case)

G2: TB no IRIS (Control)

G3: No TB or IRIS (Control)
IL-18BP
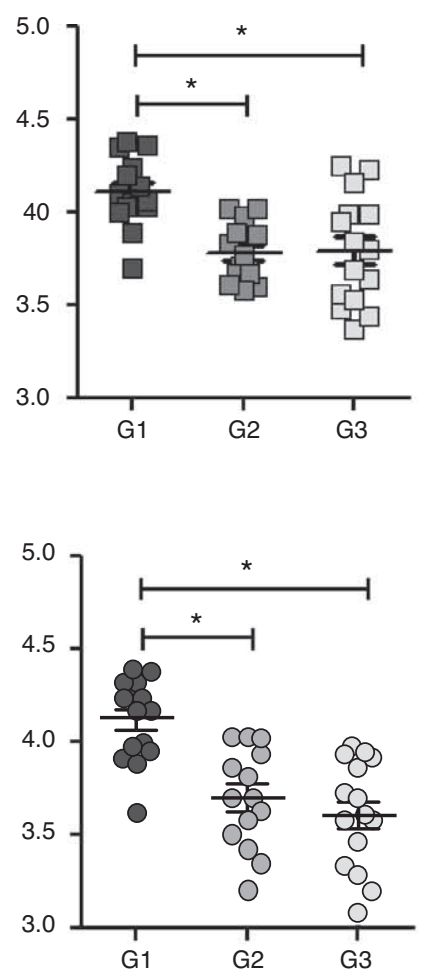

Free IL-18
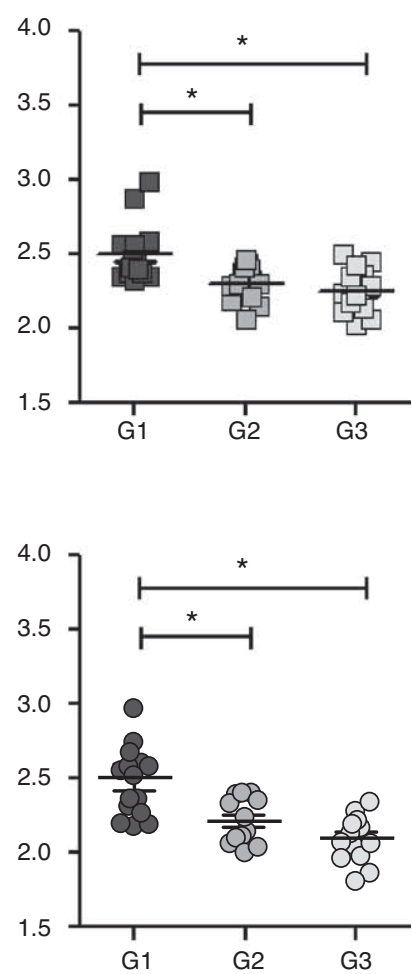

(b) ROC analysis for free IL-18

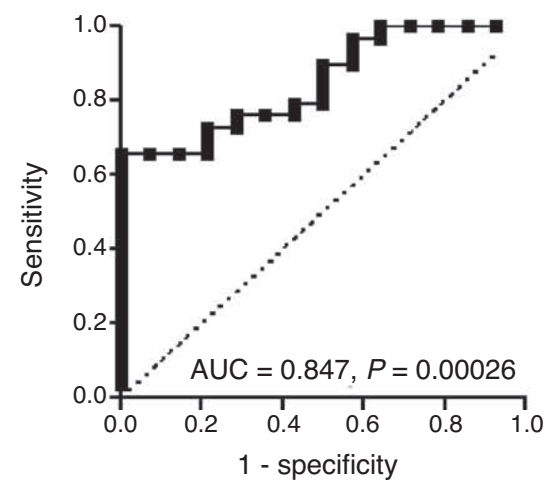

Fig. 4. Plasma levels of interleukin-18, IL-18BPa and free circulating interleukin-18 in tuberculosis-associated immune reconstitution inflammatory syndrome patients and controls. (a) Plasma levels at baseline and time of clinical event. Samples at time of TB-IRIS episode were only collected for case patients; whereas samples in the control groups were selected at an equivalent time-point (EQT) post-ART. Levels of biomarkers were compared across the three patient groups and post hoc MannWhitney $U$ tests were then performed for those biomarkers with a Kruskal-Wallis test $P$ value of $<0.05$. $* P<0.0042$ (adjusted for multiple comparisons). (b) ROC analysis for the prediction of TB-IRIS using pre-ART levels of free circulating interleukin-18. AUC, area under curve; ART, antiretroviral therapy; ROC, receiver operating characteristic; TB-IRIS, tuberculosis-associated immune reconstitution inflammatory syndrome. 
(a)

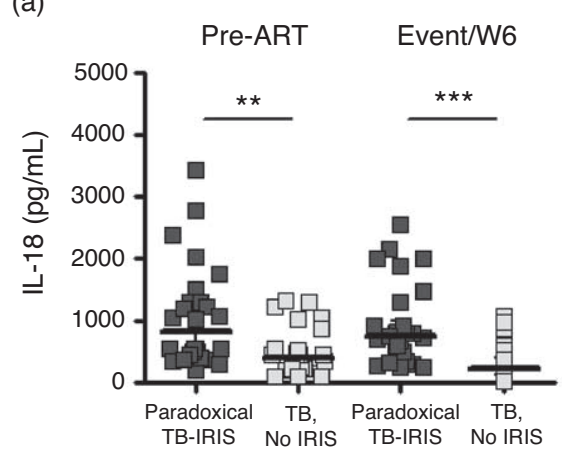

- $\operatorname{IRIS}(n=26)$

$\square$ non-IRIS $(n=22)$ (b)

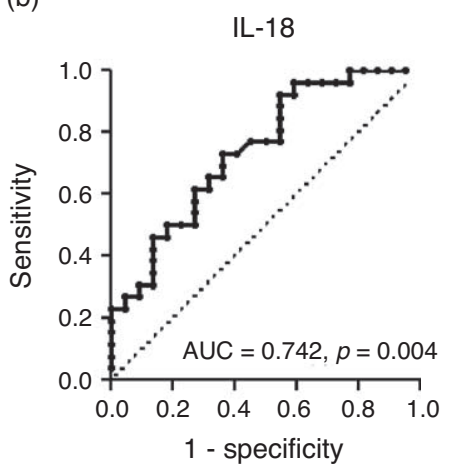

(c)

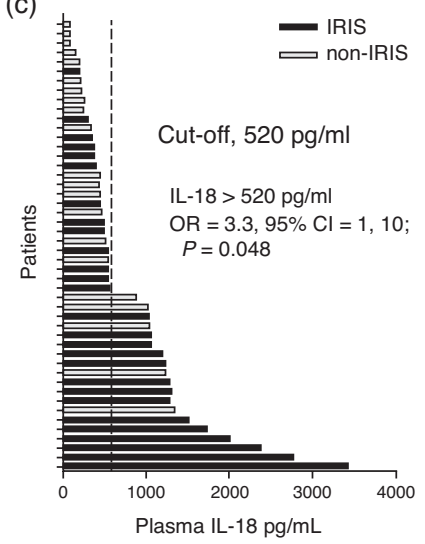

Fig. 5. Plasma levels of interleukin-18 and receiver operating characteristic analysis for Chennai cohort. (a) Comparison of plasma levels of interleukin-18 between paradoxical TB-IRIS and TB no IRIS. Samples at time of TB-IRIS episode were only collected for case patients; whereas samples in the control groups were selected at 6 weeks post-ART. (b) ROC analysis for the prediction of paradoxical TB-IRIS. (c) Cut-off value of interleukin-18 that predicts TB-IRIS. Levels of interleukin-18 was compared using Mann-Whitney $U$ test, ${ }^{*} P P<0.01,{ }^{* * *} P<0.001$. W6, 6 weeks; ART, antiretroviral therapy; AUC, area under curve; ROC, receiver operating characteristic; $\mathrm{Cl}$, confidence interval; $\mathrm{OR}$, odds ration; TB-IRIS, tuberculosis-associated immune reconstitution inflammatory syndrome.

pathways [31,32]. In the current study, we observed that HIV-TB coinfection was associated with potent monocyte/macrophage activation, as evidenced by elevation of sCD14 prior to ART initiation and during the TB-IRIS event. However, this biomarker did not appear to be as informative as interleukin-18 for predicting TB-IRIS.

Our findings confirm that plasma interleukin-18 levels are increased during TB-IRIS [14] and, furthermore, provide evidence that they predict the development of both paradoxical and unmasking TB-IRIS better than the other biomarkers examined in this study. Interleukin-18 is a potent proinflammatory cytokine predominantly produced by activated monocytes/macrophages. It was initially discovered as IFN- $\gamma$ inducing factor, and has a critical role in enhancing Th1 immune responses. Interleukin-18 is present in monocytes/macrophages as a biologically inactive precursor, prointerleukin-18, that requires further cleavage by intracellular cysteine protease caspase-1 via activation of the NLRP3 inflammasome [18]. Biologically active interleukin-18 secreted by monocytes/macrophages induces activation of nuclear factor- $\mathrm{\kappa B}$ and Fas ligand expression, as well as induction of both $\mathrm{CC}$ and $\mathrm{CXC}$ chemokines from a wide range of cells [33]. Furthermore, interleukin-18 also enhances natural killer (NK) cell cytotoxicity, neutrophil activity, and IFN- $\gamma$ production by T cells and NK cells [29].

We would, therefore, expect the elevation of interleukin18 to be accompanied by elevation of IFN- $\gamma$ among TBIRIS patients but observed that TB-IRIS was associated with lower plasma IFN- $\gamma$ levels. Various findings for serum/plasma IFN- $\gamma$ levels have been reported in different cohorts of TB-IRIS patients [22,34,35], including two cohorts of patients studied by some of the authors of this study [24]. A study of tuberculous meningitis (TBM)-IRIS patients reported similar findings to ours, whereby low cerebrospinal fluid IFN- $\gamma$ levels at TBM diagnosis predicted the development of an IRIS [36]. Data from our own studies of TB-IRIS (this study and [24]) suggest that plasma IFN- $\gamma$ levels are related to $\mathrm{CD}^{+}$T-cell counts pre-ART. Thus, patients in the Chennai cohort [24] had the highest IFN- $\gamma$ levels and a median baseline CD4 ${ }^{+}$T-cell count of approximately 200 cells/ $\mu$ l, patients in a South African cohort [24] had intermediate IFN- $\gamma$ levels and a median baseline CD $4^{+}$ T-cell count of approximately 100 cells $/ \mu 1$, and patients in the Kuala Lumpur cohort reported here had the lowest IFN- $\gamma$ levels and a median $\mathrm{CD}^{+}$T-cell count of approximately 50 cells $/ \mu 1$. We, therefore, suggest that any effect of interleukin-18 activity on plasma IFN- $\gamma$ levels is over-ridden by the effect of $\mathrm{CD}^{+}$T-cell deficiency.

Interleukin-18 activity is regulated by IL-18BP, a soluble molecule that binds mature interleukin-18 with high affinity and prevents its interaction with cell surface receptors, hence, limiting its biological activity [19]. The expression of IL-18BP is regulated by IFN- $\gamma$. The promoter region of the IL-18BP gene contains an IFN regulatory factor 1 response element that increases IL18BP gene expression upon IFN- $\gamma$ stimulation $[37,38]$. Therefore, interleukin-18 indirectly increases the production of its own inhibitor in a negative feedback loop [39]. We postulated that the inflammatory response driven by increased interleukin-18 production among TB-IRIS patients might be fuelled by a relative deficiency of IL-18BP and tested this by assaying IL-18BPa levels and calculating free circulating interleukin-18 levels in plasma. We demonstrated that free interleukin-18 levels were $40 \%$ higher in TB-IRIS patients compared with 
controls. Published data indicate that IL-18BPa is usually present with a 20 -fold molar excess over interleukin-18 in the plasma of healthy individuals [26]. Another in-vitro study showed that at least a two molar excess of IL-18BP was required to neutralize 95\% of interleukin-18 biological activity [27]. Our results, therefore, suggest that the biological activity of interleukin-18 may be inadequately regulated in patients with TB-IRIS.

We also observed increased production of CXCL10 and CXCL8 during TB-IRIS, confirming the findings of Oliver et al. [14] for CXCL10. These chemokines play an important role in the recruitment and activation of $\mathrm{T}$ cells, NK cells, and neutrophils at sites of infection [4042]. This finding is in line with histopathology findings in biopsy material, which demonstrate that TB-IRIS is associated with granulomata that exhibit a mixed inflammatory cell infiltrate [43,44]. Furthermore, TBM-IRIS patients exhibit a marked increase of cerebrospinal fluid neutrophils [36].

There are several limitations of this study. While the matching strategy led to cases and controls having similar clinical characteristics, our control groups may have been heterogeneous for non-IRIS events. Also because of the difficulties in defining latent TB infection, we do not know how many of the no TB or IRIS controls were ever exposed to $M$. tuberculosis or had resolved previous infection. Finally, patient numbers were small and, even though our findings for interleukin-18 in Malaysian patients were confirmed by similar results obtained in an independent cohort of HIV patients with paradoxical TB-IRIS from India, caution must be exercised in interpreting the findings of ROC analyses.

In summary, our data provide an important insight into the role of innate immune responses in the immunopathogenesis of TB-IRIS, particularly the role of interleukin-18, CXCL10, and CXCL8. The elevation of interleukin-18 among TB-IRIS patients raises the possibility that the pathogenesis of TB-IRIS includes an NLRP3 inflammasome disorder and, furthermore, that the biological activity of interleukin-18 may be inadequately regulated by IL-18BP. Future study of inflammasome activation and regulation in TB-IRIS is warranted. In addition, plasma levels of interleukin-18 should be validated as a biomarker of TB-IRIS, and possibly other types of IRIS, in large prospective clinical studies.

\section{Acknowledgements}

The authors thank all the study participants and all medical officers from I.D. Clinic and Unit of Medical Record Office of U.M.M.C. The authors also thank Yeat Mei Lee for her assistance in sample collection; Nazim Ahmad and Mohammad Faruq for laboratory support;
Anthony Jaworowski for providing consultations to H. Y. T.; Yong Kek Pang and Sin How Lim for critical reading for the manuscript. The authors gratefully acknowledge the contribution to this work of the Victorian Operational Infrastructure Support Program received by the Burnet Institute.

Source of funding: This work was supported by a High Impact Research grant to A.K. from the Ministry of Higher Education, Malaysia (UM.C/625/1/HIR/ MOHE/MED/01). H.Y.T. was funded by the Graduate Research Assistantship Schemes 2010-2012, University of Malaya. S.M.C. is supported by an Australian National Health and Medical Research Council (NHMRC) Principal Research Fellowship. The work of B.B.A. and I.S. is supported by the Intramural Research Program of NIAID/NIH and the study conducted on patients in the Chennai cohort was supported in part by the NIH Intramural-to-India Program 2008.

Justification of the number of contributors greater than 10: We request that 12 authors be allowed because the study has been conducted at two sites.

Data from Malaysia cohort has been presented for poster presentation at 20th International AIDS Conference, Melbourne, Australia, July 20-25, 2014. Abstract no.: A641-0064-04477.

\section{Conflicts of interest}

The authors have no conflict of interest.

\section{References}

1. Chang CC, Sheikh V, Sereti I, French MA. Immune reconstitution disorders in patients with HIV infection: from pathogenesis to prevention and treatment. Curr HIVIAIDS Rep 2014; 11:223-232.

2. Meintjes G, Lawn SD, Scano F, Maartens G, French MA, Worodria $\mathrm{W}$, et al. Tuberculosis-associated immune reconstitution inflammatory syndrome: case definitions for use in resource-limited settings. Lancet Infect Dis 2008; 8:516-523.

3. Meintjes G, Wilkinson RJ, Morroni C, Pepper DJ, Rebe K, Rangaka MX, et al. Randomized placebo-controlled trial of prednisone for paradoxical tuberculosis-associated immune reconstitution inflammatory syndrome. AIDS 2010; 24: 2381-2390.

4. Murdoch DM, Venter WD, Van Rie A, Feldman C. Immune reconstitution inflammatory syndrome (IRIS): review of common infectious manifestations and treatment options. AIDS Res Ther 2007; 4:9.

5. Grant PM, Komarow L, Andersen J, Sereti I, Pahwa S, Lederman $M M$, et al. Risk factor analyses for immune reconstitution inflammatory syndrome in a randomized study of early vs. deferred ART during an opportunistic infection. PLOS One 2010; 5:e11416.

6. Shelburne SA, Visnegarwala F, Darcourt J, Graviss EA, Giordano $\mathrm{TP}$, White $A C$ Jr, Hamill RJ. Incidence and risk factors for immune reconstitution inflammatory syndrome during highly active antiretroviral therapy. AIDS 2005; 19:399-406.

7. Breton G, Duval X, Estellat C, Poaletti X, Bonnet D, Mvondo Mvondo D, et al. Determinants of immune reconstitution inflammatory syndrome in HIV type 1-infected patients with tuberculosis after initiation of antiretroviral therapy. Clin Infect Dis 2004; 39:1709-1712. 
8. Lawn SD, Myer L, Bekker LG, Wood R. Tuberculosis-associated immune reconstitution disease: incidence, risk factors and impact in an antiretroviral treatment service in South Africa. AIDS 2007; 21:335-341.

9. Bourgarit $A$, Carcelain $G$, Martinez $V$, Lascoux C, Delcey $V$, Gicquel B, et al. Explosion of tuberculin-specific Th1-responses induces immune restoration syndrome in tuberculosis and HIV co-infected patients. AIDS 2006; 20:F1-7.

10. Antonelli LR, Mahnke Y, Hodge JN, Porter BO, Barber DL, DerSimonian $\mathrm{R}$, et al. Elevated frequencies of highly activated CD4+ T cells in HIV+ patients developing immune reconstitution inflammatory syndrome. Blood 2010; 116:38183827.

11. Mahnke YD, Greenwald JH, DerSimonian R, Roby G, Antonelli $\mathrm{LR}$, Sher $\mathrm{A}$, et al. Selective expansion of polyfunctional pathogen-specific CD4(+) T cells in HIV-1-infected patients with immune reconstitution inflammatory syndrome. Blood 2012; 119:3105-3112.

12. Tan DB, Yong YK, Tan HY, Kamarulzaman A, Tan LH, Lim A, et al. Immunological profiles of immune restoration disease presenting as mycobacterial lymphadenitis and cryptococcal meningitis. HIV Med 2008; 9:307-316.

13. Meintjes G, Wilkinson KA, Rangaka MX, Skolimowska K, van Veen $K$, Abrahams M, et al. Type 1 helper T cells and FoxP3positive T cells in HIV-tuberculosis-associated immune reconstitution inflammatory syndrome. Am / Respir Crit Care Med 2008; 178:1083-1089.

14. Oliver BG, Elliott JH, Price P, Phillips M, Saphonn V, Vun MC, et al. Mediators of innate and adaptive immune responses differentially affect immune restoration disease associated with Mycobacterium tuberculosis in HIV patients beginning antiretroviral therapy. I Infect Dis 2010; 202:1728-1737.

15. Tan DB, Lim A, Yong YK, Ponnampalavanar S, Omar S, Kamarulzaman A, et al. TLR2-induced cytokine responses may characterize HIV-infected patients experiencing mycobacterial immune restoration disease. AIDS 2011; 25:1455-1460.

16. Barber DL, Andrade BB, Sereti I, Sher A. Immune reconstitution inflammatory syndrome: the trouble with immunity when you had none. Nat Rev Microbiol 2012; 10:150-156.

17. Tran HT, Van den Bergh R, Vu TN, Laukens K, Worodria W, Loembe $M M$, et al. The role of monocytes in the development of Tuberculosis-associated Immune Reconstitution Inflammatory Syndrome. Immunobiology 2014; 219:37-44.

18. Novick D, Kim S, Kaplanski G, Dinarello CA. Interleukin18, more than a Th1 cytokine. Semin Immunol 2013; 25: 439-448.

19. Novick D, Kim SH, Fantuzzi G, Reznikov LL, Dinarello CA, Rubinstein M. Interleukin-18 binding protein: a novel modulator of the Th1 cytokine response. Immunity 1999; 10:127136.

20. $\overline{T a n} \mathrm{HY}$, Yong YK, Lim SH, Ponnampalavanar S, Omar SF, Pang $Y K$, et al. Tuberculosis (TB)-associated immune reconstitution inflammatory syndrome in TB-HIV co-infected patients in Malaysia: prevalence, risk factors, and treatment outcomes. Sex Health 2014; 11:532-539.

21. Haddow LJ, Easterbrook PJ, Mosam A, Khanyile NG, Parboosing $R$, Moodley $P$, Moosa MY. Defining immune reconstitution inflammatory syndrome: evaluation of expert opinion versus 2 case definitions in a South African cohort. Clin Infect Dis 2009; 49:1424-1432.

22. Haddow LJ, Dibben O, Moosa MY, Borrow P, Easterbrook PJ. Circulating inflammatory biomarkers can predict and characterize tuberculosis-associated immune reconstitution inflammatory syndrome. AIDS 2011; 25:1163-1174.

23. Narendran G. Comparing daily vs intermittent regimen of ATT in HIV with pulmonary tuberculosis. 2009. http://clinicaltrials.gov/ct2/show/NCTO0. [Accessed 8 August 2014].

24. Andrade BB, Singh A, Narendran G, Schechter ME, Nayak K, Subramanian S, et al. Mycobacterial antigen driven activation of CD14++CD16- monocytes is a predictor of tuberculosisassociated immune reconstitution inflammatory syndrome. PLoS Pathog 2014; 10:e1004433.
25. Narendran G, Andrade BB, Porter BO, Chandrasekhar C, Venkatesan $\mathrm{P}$, Menon PA, et al. Paradoxical tuberculosis immune reconstitution inflammatory syndrome (TB-IRIS) in HIV patients with culture confirmed pulmonary tuberculosis in India and the potential role of IL-6 in prediction. PLoS One 2013; 8:e63541.

26. Novick D, Schwartsburd B, Pinkus R, Suissa D, Belzer I, Sthoeger $Z$, et al. A novel IL-18BP ELISA shows elevated serum IL18BP in sepsis and extensive decrease of free IL-18. Cytokine 2001; 14:334-342.

27. Kim SH, Eisenstein M, Reznikov L, Fantuzzi G, Novick D, Rubinstein M, Dinarello CA. Structural requirements of six naturally occurring isoforms of the IL-18 binding protein to inhibit IL-18. Proc Natl Acad Sci U S A 2000; 97:1190-1195.

28. Migliorini P, Anzilotti C, Pratesi F, Quattroni P, Bargagna M, Dinarello CA, Boraschi D. Serum and urinary levels of IL-18 and its inhibitor IL-18BP in systemic lupus erythematosus. Eur Cytokine Netw 2010; 21:264-271.

29. Dinarello CA, Novick D, Kim S, Kaplanski G. Interleukin-18 and IL-18 binding protein. Front Immunol 2013; 4:289.

30. Serhan CN, Brain SD, Buckley CD, Gilroy DW, Haslett C, $\mathrm{O}^{\prime}$ Neill LA, et al. Resolution of inflammation: state of the art, definitions and terms. FASEB / 2007; 21:325-332.

31. Mogensen TH. Pathogen recognition and inflammatory signaling in innate immune defenses. Clin Microbiol Rev 2009; 22:240-273.

32. Schenten D, Medzhitov R. The control of adaptive immune responses by the innate immune system. Adv Immunol 2011; 109:87-124.

33. Dinarello CA, Novick D, Puren AJ, Fantuzzi G, Shapiro L, Muhl $\mathrm{H}$, et al. Overview of interleukin-18: more than an interferongamma inducing factor. I Leukoc Biol 1998; 63:658-664.

34. Conesa-Botella A, Meintjes G, Coussens AK, van der Plas $H$ Goliath R, Schutz C, et al. Corticosteroid therapy, vitamin D status, and inflammatory cytokine profile in the HIV-tuberculosis immune reconstitution inflammatory syndrome. Clin Infect Dis 2012; 55:1004-1011.

35. Tadokera R, Meintjes G, Skolimowska KH, Wilkinson KA, Matthews K, Seldon R, et al. Hypercytokinaemia accompanies HIV-tuberculosis immune reconstitution inflammatory syndrome. Eur Respir / 2011; 37:1248-1259.

36. Marais S, Meintjes G, Pepper DJ, Dodd LE, Schutz C, Ismail Z, et al. Frequency, severity, and prediction of tuberculous meningitis immune reconstitution inflammatory syndrome. Clin Infect Dis 2013; 56:450-460.

37. Muhl H, Kampfer H, Bosmann M, Frank S, Radeke H, Pfeilschifter J. Interferon-gamma mediates gene expression of IL-18 binding protein in nonleukocytic cells. Biochem Biophys Res Commun 2000; 267:960-963.

38. Hurgin V, Novick D, Rubinstein M. The promoter of IL-18 binding protein: activation by an IFN-gamma -induced complex of IFN regulatory factor 1 and CCAAT/enhancer binding protein beta. Proc Natl Acad Sci U S A 2002; 99:16957-16962.

39. Boraschi D, Dinarello CA. IL-18 in autoimmunity: review. Eur Cytokine Netw 2006; 17:224-252.

40. Christensen JE, de Lemos C, Moos T, Christensen JP, Thomsen AR. CXCL10 is the key ligand for CXCR3 on CD8+ effector T cells involved in immune surveillance of the lymphocytic choriomeningitis virus-infected central nervous system. / $/ \mathrm{m}$ munol 2006; 176:4235-4243.

41. Deshmane SL, Kremlev S, Amini S, Sawaya BE. Monocyte chemoattractant protein-1 (MCP-1): an overview. I Interferon Cytokine Res 2009; 29:313-326.

42. Hoffmann E, Dittrich-Breiholz $\mathrm{O}$, Holtmann $\mathrm{H}$, Kracht $\mathrm{M}$. Multiple control of interleukin-8 gene expression. I LeukOC Biol 2002; 72:847-855

43. Martin-Blondel G, Mars LT, Liblau RS. Pathogenesis of the immune reconstitution inflammatory syndrome in HIV-infected patients. Curr Opin Infect Dis 2012; 25:312-320.

44. Lawn SD, Wainwright $\mathrm{H}$, Orrell C. Fatal unmasking tuberculosis immune reconstitution disease with bronchiolitis obliterans organizing pneumonia: the role of macrophages. AIDS 2009; 23:143-145. 